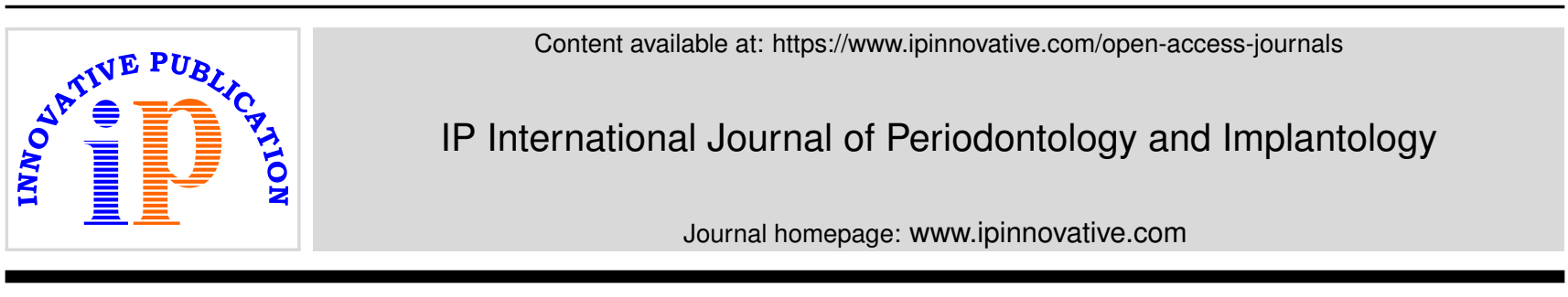

Original Research Article

\title{
Perception of general dental practitioners towards periodontal practice and referral profile: A survey study
}

\author{
Pragathi R Bhat ${ }^{1, *}$, Shivani S Desai ${ }^{1}$, Devika Padmakumar', Vijay A. Trasad ${ }^{2}$ \\ ${ }^{1}$ Dept. of Periodontics, SDM College of Dental Sciences \& Hospital, Dharwad, Karnataka, India \\ ${ }^{2}$ Dept. of Pedodontics \& Preventive Dentistry, SDM College of Dental Sciences \& Hospital, Dharwad, Karnataka, India
}

\section{A R T I C L E I N F O}

\section{Article history:}

Received 13-03-2020

Accepted 12-05-2020

Available online 18-08-2020

\section{Keywords:}

Periodontal referral

General dentists

Periodontal treatment

Dental practice

Speciality practice.

\begin{abstract}
A B S T R A C T
Context and Design: The speciality of periodontology has been evolved with leaps and bounds in all aspects ranging from advances in diagnostic aids to use of growth factors and periodontal regeneration techniques in treatment, thus making speciality per se reach new heights. Moreover, with development of technology, periodontal treatment is freely accessible to general dental practitioners. However, knowledge of periodontal diagnosis and treatment is usually limited to undergraduate level and most of times private practice deals only with chief complaint of patient.

Aim: Evaluate the knowledge and perception of general dentists towards periodontal treatment.

Materials and Methods: The study involved a survey for 50 practicing general dentists having their clinics in Hubli-Dharwad city with qualification of Bachelor of Dental Surgery only. A pre-tested close ended questionnaire was distributed comprising of 20 questions and knowledge, perception levels were assessed.

Statistical Analysis used: Percentage-wise distribution of responses to various questions was used.

Results: Although $90.4 \%$ of general dentists did believe in the success of periodontal treatment and did recommend their patients to undergo various periodontal treatment procedures, $88.5 \%$ of the dentists performed most of periodontal surgeries and phase I therapy on their own. $78 \%$ of dentists referred cases to specialist only for grafting, ridge augmentation and dental implant procedures. About $71.2 \%$ of dentists considered gingival health for prosthetic considerations and $94.2 \%$ of them did evaluate periodontal condition before referring case for orthodontic treatment. It was noted that $84.6 \%$ of dentists followed up periodontal cases as a part of maintenance phase and $57.7 \%$ of them did attend CDE program regarding consequences periodontal disease progression and its treatment outcomes in past 1 year.

Conclusions: In this study, dentists reported rendering nonsurgical and surgical periodontal therapy on a wide scale. Majority of dentists did attend CDE programs on periodontology and have gained knowledge about periodontal disease and its current treatment modalities. Although dentists do have good perception on success of periodontal treatment, majority of nonsurgical and surgical periodontal treatment was performed by general dentists on their own. Thus the need of the hour is to establish a proper periodontal referral relationship among the general dentists of Hubli-Dharwad.

Key Message: Although dentists do have good perception on success of periodontal treatment, majority of nonsurgical and surgical periodontal treatment was performed by general dentists on their own. Thus the need of the hour is to establish a proper periodontal referral relationship among the general dentists of Hubli-Dharwad city.
\end{abstract}

(C) 2020 Published by Innovative Publication. This is an open access article under the CC BY-NC license (https://creativecommons.org/licenses/by-nc/4.0/)

\footnotetext{
* Corresponding author.

E-mail address: bhat.pragathi3@gmail.com (P. R. Bhat).
}

\section{Introduction}

The treatment aspects in periodontology has evolved with the use of growth factors, periodontal regeneration techniques, modification of various flap designs and use 
of dental implants with ridge augmentation procedures thus making the speciality per se reach new heights. ${ }^{1}$ Moreover, with the development of internet and an easy access available to all periodontology journals, periodontal treatment is freely accessible to general dental practitioners. However, knowledge of periodontal diagnosis and treatment is usually limited to undergraduate level and most of times private practice deals only with chief complaint of patient. ${ }^{1}$ When it comes to periodontal disease, most of its symptoms are noticed at the later stages and its usually then that the general dentists refer the case to a periodontist. ${ }^{2}$ Thus the need of the hour is the early recognition of periodontal disease by the general dentist and basic periodontal examination be mandatory to all the teeth as a part of the routine dental checkup and refer the case to the specialist. ${ }^{3}$ But it is noticed that most of the young general dentists treat periodontal cases on their own and keep the periodontal patients in their practices for years and perform repetitive sessions of soft tissue treatment on their own as opposed to having a specialist provide the treatment thus resulting in a compromised periodontium. ${ }^{4}$ Such patients when referred to a specialist are not benefited from periodontal treatment.

With the emerging concept of speciality dental practice, the patient will appreciate the knowledge of general dentist to diagnose the periodontal disease condition at an initial stage and will be more pleased to be under the specialist care. ${ }^{5}$ Moreover, the services rendered by the periodontist will be more effective with more confidence in the field of speciality thus providing more benefits and a better service to the patient. ${ }^{5,6}$

Thus this study was conducted with an aim to evaluate the knowledge and perception of general dentists towards periodontal treatment and identify the referral relationship between them.

\subsection{Subjects and Methods}

This survey study was conducted from July 2019 to August 2019 with the questionnaire distributed to all the practicing general dentists of Hubli-Dharwad. The survey included the sample of 50 general dental practitioners with the qualification of Bachelor of Dental Surgery only and an experience of atleast 1-year in private practice setup. A well-structured pretested questionnaire which comprised of 20 questions was distributed wherein the questions were designed to assess the knowledge and perception of general dentists towards periodontal treatment and identify the referral relationship between them. It was a closeended questionnaire with responses recorded as Yes or No. However, among the participants who enrolled in this study, no questions were left unanswered. The reliability of the questionnaire was checked with the pretested questionnaire.

\subsection{Statistical analysis}

The results were subjected to statistical analysis and a percentage-wise distribution of the responses to various questions was used.

\section{Results}

The present survey gives information about the knowledge and perception of general dentists towards periodontal treatment and identify the referral relationship between them. Out of the 50 general dental practitioners who participated in this study, $90.4 \%$ (47) of general dentists did believe in the success of periodontal treatment and did recommend their patients to undergo various periodontal treatment procedures. However, $88.5 \%$ (46) of the dentists performed most of periodontal surgeries like curettage, depigmentation procedures, flap surgeries, frenectomy, gingivectomy, crown-lengthening and phase I therapy on their own and $78 \%$ (41) of dentists referred cases to specialist only for grafting, ridge augmentation and dental implant procedures. So the present survey indicates that the role of a periodontist in private dental clinics in HubliDharwad city was chiefly limited to major periodontal and implant treatment procedures. It was observed that about $71.2 \%$ (37) of dentists considered gingival health for prosthetic considerations and $94.2 \%$ (49) of them did evaluate periodontal condition before referring the case for orthodontic treatment. It was also noted that $84.6 \%$ (44) of dentists followed up periodontal cases as a part of maintenance phase and $57.7 \%$ (30) of them did attend CDE program regarding periodontal disease progression, its consequences and treatment outcomes at various stages of disease in past 1 year.(Table 1)

\section{Discussion}

In the recent past, the concept of speciality dental practice has been routinely implemented with the developing triad made by the referral doctor, referral patient and the specialist; and the success of this triad depends upon the understanding and respect among the team members. ${ }^{7}$ Unlike other dental specialities like Orthodontia, Pedodontia or Prosthodontics, the symptoms of periodontal disease clinically manifest at the advanced stages of destruction and most of the times private practice deals only with chief complaint of patient. ${ }^{2}$ Thus the need of the hour is the early recognition of periodontal disease by the general dentist and basic periodontal examination be mandatory to all the teeth as a part of the routine dental checkup - irrespective of patient's chief complaint and refer the case to the specialist. ${ }^{2,3}$ But it is noticed that most of the young general dentists treat periodontal cases on their own and perform repetitive sessions of soft tissue treatment as opposed to having a specialist provide the treatment thus resulting in a compromised periodontium. 
Table 1: Percentage-wise distribution of study participants based on their responses to the questions

\begin{tabular}{|c|c|c|c|}
\hline $\begin{array}{c}\text { Question } \\
\text { number }\end{array}$ & Options & Frequency & Percent \\
\hline \multirow{2}{*}{1} & Yes & 50 & 96.2 \\
\hline & No & 2 & 3.8 \\
\hline \multirow{2}{*}{2} & Yes & 50 & 96.2 \\
\hline & No & 2 & 3.8 \\
\hline \multirow{2}{*}{3} & Yes & 49 & 94.2 \\
\hline & No & 3 & 5.8 \\
\hline \multirow{2}{*}{4} & Yes & 25 & 48.1 \\
\hline & No & 27 & 51.9 \\
\hline \multirow{2}{*}{5} & Yes & 44 & 84.6 \\
\hline & No & 8 & 15.4 \\
\hline \multirow{2}{*}{6} & Yes & 51 & 98.1 \\
\hline & No & 1 & 1.9 \\
\hline \multirow{2}{*}{7} & Yes & 46 & 88.5 \\
\hline & No & 6 & 11.5 \\
\hline \multirow{2}{*}{8} & Yes & 47 & 90.4 \\
\hline & No & 5 & 9.6 \\
\hline \multirow{2}{*}{9} & Yes & 47 & 90.4 \\
\hline & No & 5 & 9.6 \\
\hline \multirow{2}{*}{10} & Yes & 12 & 23.1 \\
\hline & No & 40 & 76.9 \\
\hline \multirow{2}{*}{11} & Yes & 41 & 78.8 \\
\hline & No & 11 & 21.2 \\
\hline \multirow{2}{*}{12} & Yes & 1 & 1.9 \\
\hline & No & 51 & 98.1 \\
\hline \multirow{2}{*}{13} & Yes & 16 & 30.8 \\
\hline & No & 36 & 69.2 \\
\hline \multirow{2}{*}{14} & Yes & 31 & 59.6 \\
\hline & No & 21 & 40.4 \\
\hline \multirow{2}{*}{15} & Yes & 44 & 84.6 \\
\hline & No & 8 & 15.4 \\
\hline \multirow{2}{*}{16} & Yes & 37 & 71.2 \\
\hline & No & 15 & 28.8 \\
\hline \multirow{2}{*}{17} & Yes & 49 & 94.2 \\
\hline & No & 3 & 5.8 \\
\hline \multirow{2}{*}{18} & Yes & 50 & 96.2 \\
\hline & No & 2 & 3.8 \\
\hline \multirow{2}{*}{19} & Yes & 44 & 84.6 \\
\hline & No & 8 & 15.4 \\
\hline \multirow{2}{*}{20} & Yes & 30 & 57.7 \\
\hline & No & 22 & 42.3 \\
\hline
\end{tabular}

Such patients when referred to a specialist are not benefited from periodontal treatment. ${ }^{4}$ Thus the present survey study did evaluate the perception of general dentists towards periodontal treatment and identify the referral relationship between them.

In the present study, it was noticed that $90.4 \%$ (47) of general dentists did believe in the success of periodontal treatment and did recommend their patients to undergo various periodontal treatment procedures out of which $88.5 \%$ (46) of the dentists performed most of the periodontal surgeries like curettage, depigmentation procedures, flap surgeries, frenectomy, gingivectomy, crown-lengthening and phase I therapy on their own. This could be attributed to the adequate periodontal education gained by the dental practitioners at their BDS courses. ${ }^{1}$ However dental students at an undergraduate level receive only a limited educational background and exposure to surgical skills especially in cases demonstrating advanced periodontal destruction and high periods of risk factors. ${ }^{8}$ According to Mali et $\mathrm{al}^{6}$ periodontal intervention in severe forms of disease would only give unpredictable results. Thus the need of the hour is to establish a referral trend by the general dentist to a specialist at the initial stages of periodontal disease as the services rendered by the periodontist will be more effective and beneficial to the patient. ${ }^{5,6}$ According to Cherian DA et al (2019) ${ }^{3}$ with the emerging concept of speciality dental practice, the patient will appreciate the knowledge of general dentist to diagnose the periodontal disease condition at an initial stage and will be more pleased to be under the specialist care. However, $78 \%$ (41) of dentists referred cases to specialist only for grafting, ridge augmentation and dental implant procedures. This is in accordance with the factors associated with dentists' adoption of major periodontal treatment and implant therapy into practice. $^{9}$

In the present study, it was observed that majority of the general dentists practicing in Hubli-Dharwad did consider the periodontal status before providing interdisciplinary treatment, more than half of the dentists followed up periodontal cases as a part of maintenance phase and a few of the general dentists did attend the CDE program regarding periodontal disease progression, its consequences and treatment outcomes in the past 1 year - all of which revealed that the dental practitioners in HubliDharwad city did have adequate knowledge and positive perception towards periodontal treatment. However, it was noticed that 46 out of the 50 general dentists performed most of the periodontal surgeries like curettage, depigmentation procedures, flap surgeries, frenectomy, gingivectomy, crown-lengthening and phase I therapy on their own while $78 \%$ of dentists referred cases only for periodontal procedures associated with grafting, ridge augmentation and implant treatment which suggests that although patient referral system to the specialist is being implemented by the general dentists in hubli-dharwad city, it is only for major periodontal surgeries or severe forms of periodontal diseases.

Cherian DA et al $(2019)^{3}$ suggested that patients when referred to the specialist at the worsening periodontal condition may not be benefited from the services of the specialist; also the services of the periodontist could be underestimated by the patient and the general dentist. So the need of the hour is to establish the referral trend to the specialist at the initial stage of periodontal disease so as to render a better and an effective treatment to the patient. ${ }^{3}$ 
In the present study, only 30 out of the 50 general dental practitioners did attend the CDE program regarding periodontal disease and treatment outcomes at different stages of disease in the past 1 year. According to Lee JH (2009) ${ }^{4}$, dentists who did not refer patients to the periodontist were less interested in attending CDE programs in periodontics than dentists who made such referrals, which is in accordance to the results of the present study where majority of the dentists performed most of the surgical periodontal treatment and phase I therapy on their own. According to Ghiabi and Mattews (2010), ${ }^{7}$ factors affecting the patient referral protocol to the specialist depends upon the proximity to a periodontist, interpersonal relationship between the dentist and the specialist, patient's experience and positive feedback about the treatment rendered by the periodontist and success of the periodontal treatment performed by the specialist in the past. ${ }^{4,7,10}$ So the present survey indicates that the role of a periodontist in private dental clinics in Hubli-Dharwad city was chiefly limited to major periodontal and implant treatment procedures.

\section{Conclusion}

The present survey study showed an overall positive perception of general dentists towards periodontal treatment. However, the role of a periodontist was chiefly limited to major periodontal and implant treatment procedures. Thus more CDE programs on periodontal disease progression, its consequences and treatment outcomes at various stages of disease should be conducted in Hubli-Dharwad city so as to change the referral pattern of rendering periodontal treatment by the general dentists.

\section{Acknowledgements}

1. The authors wish to acknowledge Dr. Kriti Nikhil for statistical analysis and interpretation of the data.

2. The authors also acknowledge all the general dental practitioners for their time and being a part of this study.

\section{Source of Funding}

None.

\section{Conflict of Interest}

None.

\section{References}

1. Glicksman MA. Referral of the periodontal patient to the periodontist. Periodontol 2000. 2001;25(1):110-3.

2. Neuman T, Kokkeloid C. Textbook of Clinical Periodontology. Carranza Elsevier publication; 2012. p. 982.

3. Cherian D, Dayakar MM, Thermadam T. Rationale of referral of patients to a periodontist by general practitioners: Review with a crosssectional survey. J Interdiscip Dent. 2015;5(1):7-11.

4. Lee JH, Bennett DE, Richards PS, Inglehart MR. Periodontal Referral Patterns of General Dentists: Lessons for Dental Education. J Dent Educ . 2009;73(2):199-210.

5. McGuire MK, Scheyer ET. A Referral-Based Periodontal Practice -Yesterday, Today, and Tomorrow. J Periodontol. 2003;74(10):15424

6. Mali A, Mali R, Mehta H. Perception of general dental practitioners toward periodontal treatment: A survey. J Indian Soc Periodontol. 2008;12(1):4-7.

7. Ghiabi E, Matthews DC. Periodontal practice and referral profile of general dentists in Nova Scotia Canada. J Can Dent Assoc. 2012;78:c55.

8. Cobb CM, Carrara A, El-Annan E, Youngblood LA, Becker BE, Becker W, et al. Periodontal referral patterns. J Periodontol 1980;74:1470-4.

9. Esfandiari S, Majdzadeh R, Feine J. Types of Canadian Dentists Who Are More Likely to Provide Dental Implant Treatment. Implant Dent. 2011;20(1):76-84.

10. Lanning SK, Best AM, Hunt RJ. Periodontal Services Rendered by General Practitioners. J Periodontol. 2007;78(5):823-32.

\section{Author biography}

Pragathi R Bhat Assistant Professor

Shivani S Desai House Surgeon

Devika Padmakumar House Surgeon

Vijay A. Trasad Associate Professor

Cite this article: Bhat PR, Desai SS, Padmakumar D, Trasad VA Perception of general dental practitioners towards periodontal practice and referral profile: A survey study. IP Int J Periodontol Implantol 2020;5(2):74-77. 